\title{
The Riesz-Kantorovich formula for lexicographically ordered spaces
}

\author{
W. M. Schouten ${ }^{1}$
}

Received: 21 August 2017 / Accepted: 21 September 2017 / Published online: 3 October 2017 C The Author(s) 2017. This article is an open access publication

\begin{abstract}
If $L$ and $M$ are partially ordered vector spaces, then one can consider regular linear maps from $L$ to $M$, i.e. linear maps which can be written as the difference of two positive linear maps. If the space $L$ is directed, then the space $L^{r}(L, M)$ of all regular linear operators becomes a partially ordered vector space itself. We will mainly concern ourselves with the questions when the space $L^{r}(L, M)$ is itself a Riesz space and how, even if it is not a Riesz space, its lattice operations work. The so-called Riesz-Kantorovich theorem gives sufficient conditions for which $L^{r}(L, M)$ is a Riesz space and it also specifies the lattice operations by means of the Riesz-Kantorovich formula: if $S, T \in L^{r}(L, M)$ and $x \in L$ with $x \geq 0$ then the supremum $S \vee T$ in the point $x$ is given by

$$
(S \vee T)(x)=\sup \{S(y)+T(x-y): 0 \leq y \leq x\}
$$

It is still an open problem if whenever in a more general setting the supremum of two regular operators exists in $L^{r}(L, M)$, it automatically is given by the RieszKantorovich formula. Our main result concerns the special case where $L$ is a partially ordered vector space with a strong order unit and $M$ is a (possibly infinite) product of copies of the real line, equipped with the lexicographic ordering. It will turn out that under some mild continuity and regularity conditions the lattice operations on $L^{r}(L, M)$ are indeed given by the Riesz-Kantorovich formula, even though the space $L^{r}(L, M)$ is not necessarily a Riesz space.
\end{abstract}

\footnotetext{
$凶$ W. M. Schouten

w.m.schouten@math.leidenuniv.nl

1 Mathematisch Instituut, Universiteit Leiden, P.O. Box 9512, 2300 RA Leiden, The Netherlands
} 
Keywords Lexicographic order · Riesz-Kantorovich formula · Partially ordered vector spaces

Mathematics Subject Classification 6.015: Ordered structures; 47.010: Special classes of linear operators

\section{Introduction}

In this section we will define the necessary terminology and we will formulate the Riesz-Kantorovich theorem. More general theory on this subject can be found for instance in $[4,7,9]$.

First we define the spaces we will be working with. Let $V$ be a real vector space and $\leq$ a partial order on $V$. Then $(V, \leq)$ is called a partially ordered vector space if for all $x, y, z \in V$ and $\lambda \in \mathbb{R}_{\geq 0}$ the inequality $x \leq y$ implies $x+z \leq y+z$ and $x \leq y$ implies $\lambda x \leq \lambda y$. If $x \in \bar{V}$ satisfies $x \geq 0$, then we say that $x$ is positive and we introduce $V_{+}$for the set of positive vectors, called the positive cone of $V$.

Any convex subset of a vector space $V$ which satisfies the properties (1), (2) and (3) above is called a convex cone in $V$. Instead of defining partially ordered vector spaces as a vector space with a suitable partial order one can also define it in terms of a convex cone: if $L$ is a convex cone in a real vector space $V$ then we define the order $\leq$ on $V$ by saying that $x \leq y$ if and only if $y-x \in L$. Then $(V, \leq)$ is a partially ordered vector space in the sense of the previous definition. This means that a partially ordered vector space is in fact completely characterized by its positive cone.

The partially ordered vector space $V$ is said to be directed if every element of $V$ can be written as the difference of two positive vectors. If $V$ is a partially ordered vector space and $U$ is a linear subspace of $V$ then we will view $U$ as a partially ordered vector space with the ordering inherited from $V$.

Given a subset $W$ of $V$ we say that a vector $x \in V$ is an upper (lower) bound for $W$ if $x$ majorizes (minorizes) all vectors in $W$. The vector $x \in V$ is called the supremum (infimum) of $W$ if it is the smallest upper (largest lower) bound of $W$ and is denoted by sup $W$ (inf $W$ ). A subset which has both an upper bound and a lower bound is called order-bounded. A partially ordered vector space $V$ for which $\sup \{x, y\}$ exists in $V$ for all $x, y \in V$ is called a Riesz space or vector lattice. We will use the following notation for the suprema and infima of finite sets

$$
\begin{aligned}
& \bigvee_{i=1}^{n} x_{i}=\sup \left\{x_{1}, \ldots, x_{n}\right\} \\
& \bigwedge_{i=1}^{n} x_{i}=\inf \left\{x_{1}, \ldots, x_{n}\right\}
\end{aligned}
$$

for $x_{1}, \ldots, x_{n} \in V$.

A partially ordered vector space $V$ is said to be Archimedean if for elements $u, v \in V$ with $n v \leq u$ for all $n \in \mathbb{Z}_{>0}$ we must have that $v \leq 0$. A partially ordered vector space $V$ is called Dedekind complete if every subset of $V$ which has an upper bound also has a supremum. A partially ordered vector space $V$ is said to have the 
Riesz decomposition property if for any $x_{1}, x_{2}, y \in V_{+}$with $y \leq x_{1}+x_{2}$ there exist $y_{1}, y_{2} \in V$ with $0 \leq y_{1} \leq x_{1}, 0 \leq y_{2} \leq x_{2}$ and $y_{1}+y_{2}=y$. Note that any Riesz space has the Riesz decomposition property.

Let $V$ and $W$ be two partially ordered vector spaces. A linear map $f: V \rightarrow W$ is called positive if $f(x) \in W_{+}$for all $x \in V_{+}$. The map $f$ is called order-bounded if $f$ carries order-bounded subsets of $V$ into order-bounded subsets of $W$. Finally, $f$ is called regular if it can be written as the difference of two positive linear maps. If the space $V$ is directed then we can define the ordering $\leq$ on the space of linear operators from $V$ to $W$, which we denote by $L(V, W)$, by saying that $f \leq g$ if and only if $g-f$ is positive. Then $L(V, W)$ with this ordering becomes a partially ordered vector space itself. Similarly, this ordering turns the space of all order-bounded operators and the space of all regular operators into partially ordered vector spaces, which we will denote by $L^{\sim}(V, W)$ and $L^{r}(V, W)$ respectively. We always have the following sequence of inclusions

$$
L^{r}(V, W) \subset L^{\sim}(V, W) \subset L(V, W) .
$$

Let $V$ be a partially ordered vector space. For any pair of vectors $x, y \in V$ we introduce the order interval $[x, y]$ as

$$
[x, y]=\{z \in V: z \geq x, z \leq y\} .
$$

An element $e \in V_{+}$is a strong order unit if for any $x \in V$ there exists a $\lambda \in \mathbb{R}_{>0}$ in such a way $x \leq \lambda e$. If $V$ has a strong order unit, then $V$ is also directed. Let $V$ be a partially ordered vector space and let $x \in V_{+}$. Then we define the ideal generated by the element $x$ to be the linear subspace $\bigcup_{n=1}^{\infty}[-n x, n x]$ and we denote this subspace by $V_{x}$. Note that $x$ is always a strong order unit in the space $V_{x}$. An order interval in $V$ is a subset of $V$ of the form $\{x \in V: a \leq x \leq b\}$ for some $a, b \in V$. If $X$ is a vector space over $\mathbb{R}$ and $A$ is a subset of $X$, then a vector $a \in A$ is called an internal point of $A$ if for each $x \in X$ there exists a $\lambda_{0} \in \mathbb{R}_{>0}$ such that $a+\lambda x \in A$ for each $-\lambda_{0} \leq \lambda \leq \lambda_{0}$.

Having introduced all the necessary preparations, we are ready to formulate the Riesz-Kantorovich theorem.

Theorem 1.1 (The Riesz-Kantorovich theorem) Let L, M be partially ordered vector spaces. Assume that $L$ has the Riesz decomposition property, that $L$ is directed and Archimedean and that $M$ is a Dedekind complete Riesz space. Then $L^{r}(L, M)$ is a Riesz space and for $T_{1}, \ldots, T_{n} \in L^{r}(L, M)$ and $x \in L_{+}$the lattice operations are given by the so-called Riesz-Kantorovich formula

$$
\left(\bigvee_{i=1}^{n} T_{i}\right)(x)=\sup \left\{\sum_{i=1}^{n} T_{i} x_{i}: 0 \leq x_{i} \leq x, \sum_{i=1}^{n} x_{i}=x\right\} .
$$

This theorem was established in the late 1930s independently by Riesz [10] and Kantorovich [8]. Our main object of interest is the following long-standing open problem. 
If for some partially ordered vector spaces $L, M$ with $L$ directed and regular operators $T_{1}, \ldots, T_{n}: L \rightarrow M$ the supremum $\bigvee_{i=1}^{n} T_{i}$ exists in the space $L^{r}(L, M)$, does it necessarily satisfy the Riesz-Kantorovich formula?

There have been many results, which give sufficient conditions for this question to be answered positively, for example the Riesz-Kantorovich theorem itself or the results from [5]. One of these results, proved by Aliprantis et al. [6, Theorem 3.3], forms the basis for the main theorem we prove in this paper.

Theorem 1.2 ([6, Theorem 3.3]) Let L be an ordered vector space with an order unit and a Hausdorff linear topology such that all order intervals are compact. If for some linear continuous functionals $f_{1}, \ldots, f_{m} \in L^{\sim}(L, \mathbb{R})$ the supremum $g=\bigvee_{i=1}^{m} f_{i}$ exists in $L^{\sim}(L, \mathbb{R})$ then $g$ satisfies the Riesz-Kantorovich formula.

An open problem closely related to the one we are considering, is the question under what conditions for the partially ordered vector spaces $L$ and $M$ the space $L^{r}(L, M)$ is itself a Riesz space. This problem has been studied more than the one on the Riesz-Kantorovich formula and a few results can be found for example in [1,2,12].

To conclude this section we want to give an example of a partially ordered vector space $L$, which does not have the Riesz decomposition property, so for which we cannot apply the Riesz-Kantorovich theorem, but which does satisfy all conditions of Theorem 1.2 and therefore also of our main theorem. It is based on [6, Example 2.3].

Example 1.3 Consider the vector space $\mathbb{R}^{3}$ with the following 'ice cream' cone:

$$
\begin{aligned}
C & =\left\{(x, y, z) \in \mathbb{R}^{3}: z \geq 0, z^{2} \geq 4\left(x^{2}+y^{2}\right)\right\} \\
& =\left\{\lambda(x, y, 2): \lambda \geq 0, x^{2}+y^{2} \leq 1\right\} .
\end{aligned}
$$

Since $C$ is a convex cone, it induces a partial order on $\mathbb{R}^{3}$, which we will denote by $\leq_{C}$, by $x \leq_{C} y$ if and only if $y-x \in C$. We equip the space with the Euclidean topology. Then we obtain the following properties:

1. $C$ is a closed cone.

2. The space $\left(\mathbb{R}^{3}, \leq_{C}\right)$ is finite dimensional and the cone is generating. Therefore it necessarily has a strong order unit.

3. The order intervals of $\left(\mathbb{R}^{3}, \leq_{C}\right)$ are closed bounded subsets of $\mathbb{R}^{3}$ and thus they are compact.

4. $\left(\mathbb{R}^{3}, \leq_{C}\right)$ does not have the Riesz decomposition property and is thus in particular is not a Riesz space (see [6, Example 2.3] for a proof).

\section{The lexicographic ordering}

In this section we give a definition of the lexicographic ordering on a product of copies of the real line and we will prove the basic results needed for the proof of our main theorem. In this formulation we will use the notion of an ordinal number, which is a concept from set theory. Our main result is already interesting for natural numbers, which are examples of ordinal numbers. Readers not interested in ordinal numbers may read the text with natural numbers in mind. 
Let $\alpha \neq 0$ be an ordinal number and set $M=\prod_{1 \leq j \leq \alpha} \mathbb{R}$. If $x \in M$ and $\beta \leq \alpha$ then we let $x^{(\beta)}$ denote the $\beta$ th coordinate of $x$ and $x^{(1), \ldots,(\beta)}$ the vector consisting of the first $\beta$ coordinates of $x$. We equip $M$ with the lexicographic ordering by saying that $x \leq y$ if and only if either $x=y$ or there exists some $\beta \leq \alpha$ in such a way that for all $\gamma<\beta$ we have $x^{(\gamma)}=y^{(\gamma)}$ and $x^{(\beta)}<y^{(\beta)}$. Note that in this way $M$ becomes a Riesz space as it is totally ordered. If $\alpha>1$ then $M$ is not Archimedean and therefore not Dedekind complete. The following key result is what distinguishes the lexicographic ordering from the standard componentwise ordering and turns out to be essential for the proof of our main theorem.

Lemma 2.1 Let $\alpha \neq 0$ be an ordinal number. Let $M=\prod_{1 \leq j \leq \alpha} \mathbb{R}$ with the lexicographic ordering. We give $M$ the product topology. Let $K \subset \bar{M}$ be non-empty and compact. Then $K$ has an order-maximum, i.e. there exists $x \in K$ such that $x \geq y$ for all $y \in K$.

Proof We show this by transfinite induction to $\alpha$. For $\alpha=1$ the result is well-known. Now let $\alpha \geq 1$ and assume each non-empty compact set in $\prod_{1 \leq j \leq \alpha} \mathbb{R}$ has an ordermaximum. Let $M=\prod_{1 \leq j \leq \alpha+1} \mathbb{R}$ and let $K \subset M$ be compact. Let $\pi_{\alpha}$ be the projection from $M$ onto its first $\alpha$ coordinates. Then we know that $\pi_{\alpha}$ is continuous. Hence $\pi_{\alpha}(K)$ is compact in $\prod_{1 \leq j \leq \alpha} \mathbb{R}$ and thus has an order-maximum, say $x$. Then $\left\{y^{(\alpha+1)}: y \in\right.$ $\left.K, \pi_{\alpha}(y)=x\right\}$ is a compact and non-empty subset of $\mathbb{R}$ and thus has an ordermaximum $y$. Then $(x, y) \in K$ and if $z \in K$ then $\pi_{\alpha} z \leq x$ and if $\pi_{\alpha} z=x$ then $z^{(\alpha+1)} \leq y$. Hence $z \leq(x, y)$. So $K$ has an order-maximum. Finally let $\alpha$ be a limit ordinal and assume that for each $\beta<\alpha$ we have that every non-empty compact subset of $\prod_{1 \leq j \leq \beta} \mathbb{R}$ has an order-maximum. Let $K \subset M$ be compact. Let $\beta<\alpha$ and let $\pi_{\beta}$ be the projection from $M$ on the first $\beta$ coordinates. As before we can let $x_{\beta} \in \pi_{\beta}(K)$ be the order-maximum. If $\gamma<\beta$ then clearly the first $\gamma$ coordinates of $x_{\beta}$ must be $x_{\gamma}$ since we use the lexicographic ordering. Hence we can let $x \in K$ be defined by $x^{(\beta)}=x_{\beta}^{(\beta)}$ and by construction we indeed have $x \in K$. Now if $z \in K$ with $z \neq x$ then we let $\beta<\alpha$ be minimal such that $z^{(\beta)} \neq x^{(\beta)}$ and thus we must have that $\pi_{\beta} z<x_{\beta}=\pi_{\beta} x$. Hence also $z<x$. So indeed $x$ is an order-maximum of $K$. So we get that for all $\alpha \geq 1$ and all compact $K$ in $\prod_{1 \leq j \leq \alpha} \mathbb{R}$ we must have that $K$ has an order-maximum.

Notation 2.2 Let $L$ be an ordered vector space. For any integer $m>0$ and $x \in L_{+}$ we define the following non-empty convex sets

$$
\mathcal{A}_{x}^{m}=\left\{\left(y_{1}, \ldots, y_{m}\right) \in L_{+}^{m}: \sum_{i=1}^{m} y_{i} \leq x\right\}
$$

and

$$
\mathcal{F}_{x}^{m}=\left\{\left(y_{1}, \ldots, y_{m}\right) \in L_{+}^{m}: \sum_{i=1}^{m} y_{i}=x\right\}
$$

If $x \in \prod_{j \in J} \mathbb{R}$ for some index set $J$ then we write $x^{(j)}$ for the $j$-th component of $x$ and similarly if $f: L \rightarrow \prod_{j \in J} \mathbb{R}$ is any map then we write $f^{(j)}$ for the map 
$f^{(j)}: L \rightarrow \mathbb{R}, x \mapsto(f(x))^{(j)}$. Similarly, if $\alpha$ is an ordinal, then for $x \in \prod_{1 \leq \beta \leq \alpha} \mathbb{R}$ and $\gamma<\alpha$ we mean by $x^{(1), \ldots,(\gamma)}$ the vector in $\prod_{1 \leq \beta \leq \gamma} \mathbb{R}$ which corresponds to the first $\gamma$ coordinates of $x$ and if $f: L \rightarrow \prod_{1 \leq \beta \leq \alpha} \mathbb{R}$ is any map and $\gamma<\alpha$ then we mean by $f^{(1), \ldots,(\gamma)}$ the map $f^{(1), \ldots,(\gamma)}: L \rightarrow \prod_{1 \leq \beta \leq \gamma} \mathbb{R}, x \mapsto(f(x))^{(1), \ldots,(\gamma)}$.

We have to prove one more lemma, which gives another essential property of the lexicographic ordering.

Lemma 2.3 Let $L$ be a directed partially ordered vector space. Let $\alpha \geq 1$ be an ordinal number and let $M=\prod_{1 \leq j \leq \alpha} \mathbb{R}$ with the lexicographic ordering. Let $f_{1}, \ldots, f_{m} \in$ $L^{\sim}(L, M)$ such that $f_{i}^{(j)}$ is regular for all $i \in\{1, \ldots, m\}$ and all $1 \leq j \leq \alpha$ and such that $g=f_{1} \vee \cdots \vee f_{m}$ exists in $L^{\sim}(L, M)$. Let $\beta<\alpha$. Then $f_{1}^{(1), \ldots,(\beta)} \vee \cdots \vee f_{m}^{(1), \ldots,(\beta)}$ exists and equals $g^{(1), \ldots,(\beta)}$.

Proof Suppose this is not the case. Then we can let $h: L \rightarrow \prod_{1 \leq j \leq \beta} \mathbb{R}$ be orderbounded and $x \in L_{+}$such that $h \geq f_{i}^{(1), \ldots,(\beta)}$ for all $1 \leq i \leq m$ and $h(x)<$ $g^{(1), \ldots,(\beta)}(x)$. By regularity we can write

$$
f_{i}^{(j)}=g_{i}^{(j)}-h_{i}^{(j)}
$$

where $g_{i}^{(j)} \geq 0, h_{i}^{(j)} \geq 0$ for $1 \leq i \leq m$ and $1 \leq j \leq \alpha$. Upon defining

$$
\begin{aligned}
h^{\prime}: L & \rightarrow M, \\
y & \mapsto\left(h^{(1)}(y), \ldots, h^{(\beta)}(y), \sum_{i=1}^{m} g_{i}^{(\beta+1)}(y), \ldots, \sum_{i=1}^{m} g_{i}^{(\alpha)}(y)\right),
\end{aligned}
$$

we note that $h^{\prime} \in L^{\sim}(L, M)$ and we see that $h^{\prime} \geq f_{i}$ for all $i \in\{1, \ldots, m\}$ since we use the lexicographic ordering on $M$ and since $h \geq f_{i}^{(1), \ldots,(\beta)}$ for all $1 \leq i \leq m$. Furthermore we see that

$$
\begin{aligned}
h^{\prime}(x) & =\left(h^{(1)}(x), \ldots, h^{(\beta)}(x), \sum_{i=1}^{m} g_{i}^{(\beta+1)}(x), \ldots, \sum_{i=1}^{m} g_{i}^{(\alpha)}(x)\right) \\
& <g(x),
\end{aligned}
$$

since $h(x)<g^{(1), \ldots,(\beta)}(x)$ and we use the lexicographic ordering on $M$. Hence $h^{\prime} \nsucceq g$ and that gives a contradiction with the definition of $g$.

\section{Main theorem}

We are ready to state and prove our main theorem, which is an improved version of Theorem 1.2. In their paper, Aliprantis, Tourky and Yannelis divide up the proof over two lemmas, Lemmas 3.1 and 3.2 and use these lemmas to prove the theorem. For our theorem, we use transfinite induction, starting with Theorem 1.2. In the successor ordinal case, we largely follow the steps Aliprantis, Tourky and Yannelis used: the 
proof is divided up in three parts. The proofs of the second and third part are almost identical to the proofs of [6, Lemma 3.2] and [6, Theorem 3.3] respectively. The first part is quite different and substantially more difficult; the first half is new and necessary to show something which is almost trivial in [6, Lemma 3.1] (namely that the intersection of the sets $Y$ and $Z$ defined below is empty), but certainly not so in this setting. To prove this, we need the full Riesz-Kantorovich formula for the smaller image space, not just the special case we are looking at in the first part. This is also the reason why we cannot separate this theorem into two lemmas and a theorem, just like Aliprantis, Tourky and Yannelis did. The second half is roughly the same as the proof of [6, Lemma 3.1]. The limit ordinal case is of course new, but is much easier than the successor ordinal case.

We remark that an example of a space which satisfies the properties of the space $L$ in Theorem 3.1, but not of the domain space in the Riesz-Kantorovich theorem, can be found in Example 1.3. Other examples of spaces which satisfy the properties of the space $L$ in Theorem 3.1 include any finite dimensional ordered vector space with a closed generating cone and bounded order intervals or any $C(K)$-space, for $K$ compact, Hausdorff and hyperstonean, which guarantees that $C(K)$ is a dual space, equipped with the weak*-topology and the pointwise ordering.

This theorem was first proven in the autor's Master Thesis, see [11].

Theorem 3.1 (Main theorem) Let $L$ be an ordered vector space with an order unit e and a Hausdorff linear topology in such a way that all order intervals are compact. Let $\alpha$ be an ordinal number with $\alpha \geq 1$ and let $M=\prod_{1 \leq j \leq \alpha} \mathbb{R}$ with the lexicographic ordering and the product topology. Assume we have $f_{1}, f_{2}, \ldots, f_{m}$ continuous, linear maps from $L$ to $M$ such that $f_{i}^{(j)}$ is regular for all $i \in\{1, \ldots, m\}$ and all ordinals $j$ with $1 \leq j \leq \alpha$ and such that the supremum $g=\bigvee_{i=1}^{m} f_{i}$ exists in $L^{\sim}(L, M)$. Then $g$ satisfies the Riesz-Kantorovich formula.

Proof We will prove the theorem by transfinite induction on $\alpha$. The case $\alpha=1$ follows directly from Theorem 1.2. So we fix $\alpha \geq 1$ and assume the statement of the theorem is true for image spaces $\mathbb{R}^{1}, \ldots, \prod_{1 \leq j \leq \alpha} \mathbb{R}$. Set $M=\prod_{1 \leq j \leq \alpha+1} \mathbb{R}$. The induction step for successor ordinals will consist of proving the following three statements:

(i) Assume we have $f_{1}, f_{2}, \ldots, f_{m}$ continuous, linear maps from $L$ to $M$ in such a way that $f_{i}^{(j)}$ is regular for all $i \in\{1, \ldots, m\}$ and all ordinals $j$ with $1 \leq j \leq \alpha$ and such that the supremum $\left(\bigvee_{i=1}^{m} f_{i}\right)^{+}$exists in $L^{\sim}(L, M)$. Then there exists some $\left(x_{1}^{*}, x_{2}^{*}, \ldots, x_{m}^{*}\right) \in \mathcal{A}_{e}^{m}$ satisfying

$$
\sum_{i=1}^{m} f_{i}\left(x_{i}^{*}\right) \geq \sum_{i=1}^{m} f_{i}\left(x_{i}\right)
$$

for each $\left(x_{1}, x_{2}, \ldots, x_{m}\right) \in \mathcal{A}_{e}^{m}$, together with the identity

$$
\left(\bigvee_{i=1}^{m} f_{i}\right)^{+}(e)=\sum_{i=1}^{m} f_{i}\left(x_{i}^{*}\right)
$$


(ii) Assume we have $f_{1}, f_{2}, \ldots, f_{m}$ continuous, linear maps from $L$ to $M$ such that $f_{i}^{(j)}$ is regular for all $i \in\{1, \ldots, m\}$ and all ordinals $j$ with $1 \leq j \leq \alpha$ and such that the supremum $\bigvee_{i=1}^{m} f_{i}$ exists in $L^{\sim}(L, M)$. Then there exists some $\left(x_{1}^{*}, x_{2}^{*}, \ldots, x_{m}^{*}\right) \in \mathcal{F}_{e}^{m}$ satisfying

$$
\sum_{i=1}^{m} f_{i}\left(x_{i}^{*}\right) \geq \sum_{i=1}^{m} f_{i}\left(x_{i}\right)
$$

for each $\left(x_{1}, x_{2}, \ldots, x_{m}\right) \in \mathcal{F}_{e}^{m}$, together with the identity

$$
\left(\bigvee_{i=1}^{m} f_{i}\right)(e)=\sum_{i=1}^{m} f_{i}\left(x_{i}^{*}\right)
$$

(iii) Assume we have $f_{1}, f_{2}, \ldots, f_{m}$ continuous, linear maps from $L$ to $M$ such that $f_{i}^{(j)}$ is regular for all $i \in\{1, \ldots, m\}$ and all ordinals $j$ with $1 \leq j \leq \alpha$ and such that the supremum $\bigvee_{i=1}^{m} f_{i}$ exists in $L^{\sim}(L, M)$. Then the Riesz-Kantorovich formula holds.

Proof of $(i)$ Since all $f_{i}$ are continuous and all order intervals in $L$ are compact, we see that $K=\left\{\sum_{i=1}^{m} f_{i}\left(x_{i}\right):\left(x_{1}, \ldots, x_{m}\right) \in \mathcal{A}_{e}^{m}\right\}$ is compact. Lemma 2.1 implies that $K$ contains an order-maximum with respect to the lexicographic ordering on $M$, which yields the first statement in (i).

We pick $\left(x_{1}^{*}, \ldots, x_{m}^{*}\right) \in \mathcal{A}_{e}^{m}$ in such a way that

$$
\sum_{i=1}^{m} f_{i}\left(x_{i}^{*}\right) \geq \sum_{i=1}^{m} f_{i}\left(x_{i}\right)
$$

for all $\left(x_{1}, \ldots, x_{m}\right) \in \mathcal{A}_{e}^{m}$.

Writing $g=\left(f_{1} \vee \cdots \vee f_{m}\right)^{+}$, the positivity of this function yields the estimate

$$
\begin{aligned}
g(e) & \geq g\left(\sum_{i=1}^{m} x_{i}^{*}\right) \\
& =\sum_{i=1}^{m} g\left(x_{i}^{*}\right) \\
& \geq \sum_{i=1}^{m} f_{i}\left(x_{i}^{*}\right) .
\end{aligned}
$$

Hence it remains to show that the reverse inequality holds.

First we assume that $f_{i}^{(\alpha+1)} \geq 0$ for all $i \in\{1, \ldots, m\}$. Lemma 2.3 implies that

$$
\bigvee_{i=1}^{m} f_{i}^{(1), \ldots,(\alpha)}=\left(\bigvee_{i=1}^{m} f_{i}\right)^{(1), \ldots,(\alpha)}
$$


since we use the lexicographic ordering on $\prod_{1 \leq j \leq \alpha} \mathbb{R}$. Upon writing

$$
h=\left(\bigvee_{i=1}^{m} f_{i}^{(1), \ldots,(\alpha)}\right)^{+}
$$

the induction hypothesis yields that $h$ is positive, linear and satisfies for $x \in L^{+}$the identity

$$
h(x)=\max \left\{\sum_{i=1}^{m} f_{i}\left(x_{i}\right)^{(1), \ldots,(\alpha)}:\left(x_{1}, \ldots, x_{m}\right) \in \mathcal{A}_{x}^{m}\right\} .
$$

Here the usual supremum is replaced by a maximum on account of the continuity of the functionals $f_{i}^{(1), \ldots,(\alpha)}$ and the compactness of order intervals. Then our choice of $x_{1}^{*}, \ldots, x_{m}^{*}$ yields the identity

$$
\begin{aligned}
h(e) & =\sum_{i=1}^{m} f_{i}\left(x_{i}^{*}\right)^{(1), \ldots,(\alpha)} \\
& =h\left(\sum_{i=1}^{m} x_{i}^{*}\right)
\end{aligned}
$$

To aid us in our calculations, we start by looking at the case where $\sum_{i=1}^{m} f_{i}\left(x_{i}^{*}\right)^{(\alpha+1)}$ $=0$. Seeking a contradiction, we pick $j \in\{1, \ldots, m\}$ and $y \geq 0$ and we suppose that they satisfy the identities

$$
\begin{aligned}
& h(y)=f_{j}(y)^{(1), \ldots,(\alpha)} \\
& f_{j}(y)^{(\alpha+1)}>0 .
\end{aligned}
$$

Since the defining property (3.9) of the function $h$ is given by a maximum, we can pick $z_{1}, \ldots, z_{m} \in L_{+}$in such a way that we have the identities

$$
\begin{aligned}
\sum_{i=1}^{m} z_{i} & \leq e-y \\
h(e-y) & =\sum_{i=1}^{m} f_{i}\left(z_{i}\right)^{(1), \ldots,(\alpha)} .
\end{aligned}
$$

This yields that $y+\sum_{i=1}^{m} z_{i} \leq e, y+z_{j} \geq 0, z_{i} \geq 0$ for all $i \in\{1, \ldots, m\}$, which allows us to compute

$$
\begin{aligned}
\sum_{i=1, i \neq j}^{m} f_{i}\left(z_{i}\right)^{(1), \ldots,(\alpha)}+f_{j}\left(y+z_{j}\right)^{(1), \ldots,(\alpha)} & =f_{j}(y)^{(1), \ldots,(\alpha)}+\sum_{i=1}^{m} f_{i}\left(z_{i}\right)^{(1), \ldots,(\alpha)} \\
& =h(y)+h(e-y) \\
& =h(e) \\
& =\sum_{i=1}^{m} f_{i}\left(x_{i}^{*}\right)^{(1), \ldots,(\alpha)}
\end{aligned}
$$


In addition, the positivity of the functions $f_{i}^{(\alpha+1)}$ for $i \in\{1, \ldots, m\}$ yields

$$
\begin{aligned}
\sum_{i=1, i \neq j}^{m} f_{i}\left(z_{i}\right)^{(\alpha+1)}+f_{j}\left(y+z_{j}\right)^{(\alpha+1)} & \geq f_{j}(y)^{(\alpha+1)} \\
& >0 \\
& =\sum_{i=1}^{m} f_{i}\left(x_{i}^{*}\right)^{(\alpha+1)} .
\end{aligned}
$$

So we obtain $\sum_{i=1, i \neq j}^{m} f_{i}\left(z_{i}\right)+f_{j}\left(y+z_{j}\right)>\sum_{i=1}^{m} f_{i}\left(x_{i}^{*}\right)$, which gives a contradiction with the defining property (3.5) of $\left(x_{1}^{*}, \ldots, x_{m}^{*}\right)$. Therefore for all $j \in\{1, \ldots, m\}$ and $y \in L_{+}$with $h(y)=f_{j}(y)^{(1), \ldots,(\alpha)}$ we have $f_{j}(y)^{(\alpha+1)}=0$. Clearly we also have for all $j \in\{1, \ldots, m\}$ and $y \in L_{+}$that $h(y) \geq f_{j}(y)^{(1), \ldots,(\alpha)}$ by our definition of $h$. Hence we see that for all $j \in\{1, \ldots, m\}$ and all $y \in L_{+}$we have

$$
f_{j}(y) \leq(h(y), 0)
$$

which yields

$$
f_{j} \leq(h, 0) \text {. }
$$

This implies that $g \leq(h, 0)$. In particular, we can estimate

$$
\begin{aligned}
g(e) & \leq(h(e), 0) \\
& =\left(\sum_{i=1}^{m} f_{i}\left(x_{i}^{*}\right)^{(1), \ldots,(\alpha)}, \sum_{i=1}^{m} f_{i}\left(x_{i}^{*}\right)^{(\alpha+1)}\right) \\
& =\sum_{i=1}^{m} f_{i}\left(x_{i}^{*}\right) .
\end{aligned}
$$

Therefore we obtain

$$
g(e)=\sum_{i=1}^{m} f_{i}\left(x_{i}^{*}\right)
$$

It remains to consider the case where $\sum_{i=1}^{m} f_{i}\left(x_{i}^{*}\right)^{(\alpha+1)}>0$. We introduce the set

$$
\begin{gathered}
Y=\left\{\left(y_{1}, \ldots, y_{m}\right) \in L_{+}^{m}: h\left(\sum_{i=1}^{m} y_{i}\right)=\sum_{i=1}^{m} f_{i}\left(y_{i}\right)^{(1), \ldots,(\alpha)},\right. \\
\left.\sum_{i=1}^{m} f_{i}\left(y_{i}\right)^{(\alpha+1)}>\sum_{i=1}^{m} f_{i}\left(x_{i}^{*}\right)^{(\alpha+1)}\right\} .
\end{gathered}
$$

This set is clearly convex and non-empty, as it contains $t\left(x_{1}^{*}, \ldots, x_{m}^{*}\right)$ for all $t>1$. In addition, we introduce the set

$$
Z=\left\{\left(z_{1}, \ldots, z_{m}\right) \in L^{m}: \sum_{i=1}^{m} z_{i} \leq e\right\},
$$


which is also non-empty and convex. Our goal is to that $Y$ and $Z$ are disjoint. Seeking a contradiction, we suppose that $Y \cap Z \neq \varnothing$ and pick any $\left(y_{1}, \ldots, y_{m}\right) \in Y \cap Z$. Since the defining property (3.9) of the function $h$ is given by a maximum and $e-\sum_{i=1}^{m} y_{i} \geq 0$, we can pick $z_{1}, \ldots, z_{m} \in L_{+}$in such a way that

$$
\begin{array}{ll}
\sum_{i=1}^{m} z_{i} & \leq e-\sum_{i=1}^{m} y_{i}, \\
h\left(e-\sum_{i=1}^{m} y_{i}\right) & =\sum_{i=1}^{m} f_{i}\left(z_{i}\right)^{(1), \ldots,(\alpha)} .
\end{array}
$$

This yields $\sum_{i=1}^{m}\left(y_{i}+z_{i}\right) \leq e, y_{i}+z_{i} \geq 0$ for all $i \in\{1, \ldots, m\}$, which allows us to compute

$$
\begin{aligned}
\sum_{i=1}^{m} f_{i}\left(y_{i}+z_{i}\right)^{(1), \ldots,(\alpha)} & =\sum_{i=1}^{m} f_{i}\left(y_{i}\right)^{(1), \ldots,(\alpha)}+\sum_{i=1}^{m} f_{i}\left(z_{i}\right)^{(1), \ldots,(\alpha)} \\
& =h\left(\sum_{i=1}^{m} y_{i}\right)+h\left(e-\sum_{i=1}^{m} y_{i}\right) \\
& =h(e) \\
& =\sum_{i=1}^{m} f_{i}\left(x_{i}^{*}\right)^{(1), \ldots,(\alpha)}
\end{aligned}
$$

In addition, the positivity of $f_{i}^{(\alpha+1)}$ for $i \in\{1, \ldots, m\}$ also yields

$$
\begin{aligned}
\sum_{i=1}^{m} f_{i}\left(y_{i}+z_{i}\right)^{(\alpha+1)} & \geq \sum_{i=1}^{m} f_{i}\left(y_{i}\right)^{(\alpha+1)} \\
& >\sum_{i=1}^{m} f_{i}\left(x_{i}^{*}\right)^{(\alpha+1)} .
\end{aligned}
$$

So we obtain that $\sum_{i=1}^{m} f_{i}\left(y_{i}+z_{i}\right)>\sum_{i=1}^{m} f_{i}\left(x_{i}^{*}\right)$, which gives a contradiction with the defining property (3.5) of $\left(x_{1}^{*}, \ldots, x_{m}^{*}\right)$. So we must have that $Y \cap Z=\emptyset$.

The set $Z$ has an internal point in $L^{m}$ by [6, Lemma 2.1]. On account of the separation theorem [3, Theorem 5.61], we pick a non-zero linear functional $\left(h_{1}^{(\alpha+1)}, \ldots, h_{m}^{(\alpha+1)}\right) \in\left(L^{*}\right)^{m}$ that separates $Z$ and $Y$, i.e.

$$
\sum_{i=1}^{m} h_{i}^{(\alpha+1)}\left(y_{i}\right) \geq \sum_{i=1}^{m} h_{i}^{(\alpha+1)}\left(z_{i}\right) \text { for all }\left(y_{1}, \ldots, y_{m}\right) \in Y \text { and }\left(z_{1}, \ldots, z_{m}\right) \in Z \text {. }
$$

Since $\left(x_{1}^{*}, \ldots, x_{m}^{*}\right) \in Z$ the bound (3.24) yields

$$
\sum_{i=1}^{m} h_{i}^{(\alpha+1)}\left(y_{i}\right) \geq \sum_{i=1}^{m} h_{i}^{(\alpha+1)}\left(x_{i}^{*}\right) \text { for all }\left(y_{1}, \ldots, y_{m}\right) \in Y,
$$


together with

$$
\begin{aligned}
\sum_{i=1}^{m} h_{i}^{(\alpha+1)}\left(x_{i}^{*}\right) & =\lim _{\beta \downarrow 1} \sum_{i=1}^{m} h_{i}^{(\alpha+1)}\left(\beta x_{i}^{*}\right) \\
& \geq \sum_{i=1}^{m} h_{i}^{(\alpha+1)}\left(z_{i}\right) \quad \text { for all }\left(z_{1}, \ldots, z_{m}\right) \in Z .
\end{aligned}
$$

Next, we show that $h_{1}^{(\alpha+1)}=\cdots=h_{m}^{(\alpha+1)}:=h^{(\alpha+1)}$. Suppose, by way of contradiction, that there exists some $z \in L$ such that $h_{1}^{(\alpha+1)}(z)>h_{2}^{(\alpha+1)}(z)$. Then for some $\beta>1$ we must have

$$
h_{1}^{(\alpha+1)}\left(x_{1}^{*}+z\right)+h_{2}^{(\alpha+1)}\left(x_{2}^{*}-z\right)+\sum_{i=3}^{m} h_{i}^{(\alpha+1)}\left(x_{i}^{*}\right)>\sum_{i=1}^{m} h_{i}^{(\alpha+1)}\left(\beta x_{i}^{*}\right) .
$$

However, we see that $\left(\beta x_{1}^{*}, \ldots, \beta x_{m}^{*}\right) \in Y$ since $\beta>1$ and $\left(x_{1}^{*}+z, x_{2}^{*}-\right.$ $\left.z, x_{3}^{*}, \ldots, x_{m}^{*}\right) \in Z$, which contradicts (3.24). By the symmetry of the situation we see that $h_{1}^{(\alpha+1)}=h_{2}^{(\alpha+1)}=\cdots=h_{m}^{(\alpha+1)}:=h^{(\alpha+1)}$.

Now we show that $h^{(\alpha+1)} \geq 0$. Fix $x \in L^{+}$. Note that $(e-\beta x, 0,0, \ldots) \in Z$ for all $\beta>0$. Therefore, (3.26) yields

$$
\sum_{i=1}^{m} h^{(\alpha+1)}\left(x_{i}^{*}\right) \geq h^{(\alpha+1)}(e)-\beta h^{(\alpha+1)}(x),
$$

which can be rewritten to obtain the bound

$$
h^{(\alpha+1)}(x) \geq \frac{h^{(\alpha+1)}(e)-\sum_{i=1}^{m} h^{(\alpha+1)}\left(x_{i}^{*}\right)}{\beta}
$$

for each $\beta>0$. Letting $\beta \rightarrow \infty$ yields $h^{(\alpha+1)}(x) \geq 0$. Hence $h^{(\alpha+1)} \geq 0$ and thus $h^{(\alpha+1)} \in L^{\sim}$.

Since $(e, 0, \ldots, 0) \in Z$ and $\sum_{i=1}^{m} x_{i}^{*} \leq e$ we see from Eq. (3.26) that

$$
\begin{aligned}
h^{(\alpha+1)}\left(\sum_{i=1}^{m} x_{i}^{*}\right) & \leq h^{(\alpha+1)}(e) \\
& \leq h^{(\alpha+1)}\left(\sum_{i=1}^{m} x_{i}^{*}\right)
\end{aligned}
$$

and thus we obtain the identity

$$
h^{(\alpha+1)}\left(\sum_{i=1}^{m} x_{i}^{*}\right)=h^{(\alpha+1)}(e) .
$$

Furthermore, since $h^{(\alpha+1)} \neq 0$ and $e$ is an order unit, it follows that $h^{(\alpha+1)}(e)>0$. Since $(e, 0, \ldots, 0) \in Z$ the bound (3.26) yields that $\sum_{i=1}^{m} h^{(\alpha+1)}\left(x_{i}^{*}\right)>0$. These considerations allow us to define 


$$
\delta^{(\alpha+1)}=\frac{\sum_{i=1}^{m} f_{i}\left(x_{i}^{*}\right)^{(\alpha+1)}}{\sum_{i=1}^{m} h^{(\alpha+1)}\left(x_{i}^{*}\right)} \in \mathbb{R}_{>0} .
$$

We claim that $\delta^{(\alpha+1)} h^{(\alpha+1)}(x) \geq f_{i}^{(\alpha+1)}(x)$ for each $i \in\{1, \ldots, m\}$ and $x \in L^{+}$that have $h(x)=f_{i}(x)^{(1), \ldots,(\alpha)}$. To see this, fix $i \in\{1, \ldots, m\}$ and $x \in L_{+}$in such a way that $h(x)=f_{i}(x)^{(1), \ldots,(\alpha)}$. If $f_{i}(x)^{(\alpha+1)} \leq 0$ then $\delta^{(\alpha+1)} h^{(\alpha+1)}(x) \geq 0 \geq f_{i}(x)^{(\alpha+1)}$ is trivially true. Assume, therefore, that $\overline{f_{i}}(x)^{(\alpha+1)}>0$ and define

$$
\gamma^{(\alpha+1)}=\frac{\sum_{i=1}^{m} f_{i}\left(x_{i}^{*}\right)^{(\alpha+1)}}{f_{i}(x)^{(\alpha+1)}} \in \mathbb{R}_{>0}
$$

For $\beta>1$ we can compute

$$
\begin{aligned}
f_{i}\left(\beta \gamma^{(\alpha+1)} x\right)^{(\alpha+1)} & =\beta \frac{\sum_{i=1}^{m} f_{i}\left(x_{i}^{*}\right)^{(\alpha+1)}}{f_{i}(x)^{(\alpha+1)}} f_{i}(x)^{(\alpha+1)} \\
& =\beta \sum_{i=1}^{m} f_{i}\left(x_{i}^{*}\right)^{(\alpha+1)} \\
& >\sum_{i=1}^{m} f_{i}\left(x_{i}^{*}\right)^{(\alpha+1)}
\end{aligned}
$$

since we assumed that $\sum_{i=1}^{m} f_{i}\left(x^{*}\right)^{(\alpha+1)}>0$. Hence the linearity of $h$ and $f_{i}^{(1), \ldots,(\alpha)}$ yields that $\left(0, \ldots, 0, \beta \gamma^{(\alpha+1)} x, 0, \ldots, 0\right) \in Y$, where $\beta \gamma^{(\alpha+1)} x$ takes the $i$-th position of this vector. Hence with Eq. (3.25) we get

$$
\begin{aligned}
\delta^{(\alpha+1)} \gamma^{(\alpha+1)} h^{(\alpha+1)}(x) & =\delta^{(\alpha+1)} \lim _{\beta \downarrow 1} h^{(\alpha+1)}\left(\beta \gamma^{(\alpha+1)} x\right) \\
& \geq \delta^{(\alpha+1)} \sum_{i=1}^{m} h^{(\alpha+1)}\left(x_{i}^{*}\right) \\
& =\sum_{i=1}^{m} f_{i}\left(x_{i}^{*}\right)^{(\alpha+1)} \\
& =\gamma^{(\alpha+1)} f_{i}(x)^{(\alpha+1)} .
\end{aligned}
$$

Therefore $\delta^{(\alpha+1)} h^{(\alpha+1)}(x) \geq f_{i}(x)^{(\alpha+1)}$ for all $x \in L_{+}$with $h(x)=f_{i}(x)^{(1), \ldots,(\alpha)}$. Thus, due to the lexicographic ordering on $M$, we have for all $x \geq 0$ that

$$
f_{i}(x) \leq\left(h(x), \delta^{(\alpha+1)} h^{(\alpha+1)}(x)\right)
$$

Since this bound holds for all $i \in\{1, \ldots, m\}$, we get that

$$
g(x) \leq\left(h(x), \delta^{(\alpha+1)} h^{(\alpha+1)}(x)\right)
$$

for all $x \geq 0$. In particular, we get

$$
\begin{aligned}
g(e) & \leq\left(h(e), \delta^{(\alpha+1)} h^{(\alpha+1)}(e)\right) \\
& =\sum_{i=1}^{m} f_{i}\left(x_{i}^{*}\right)
\end{aligned}
$$

since $h(e)=h\left(\sum_{i=1}^{m} x_{i}^{*}\right)$ and $h^{(\alpha+1)}(e)=h^{(\alpha+1)}\left(\sum_{i=1}^{m} x_{i}^{*}\right)$. Therefore we conclude that

$$
g(e)=\sum_{i=1}^{m} f_{i}\left(x_{i}^{*}\right)
$$


From now on, we do not assume that $f_{i}^{(\alpha+1)} \geq 0$ for $i \in\{1, \ldots, m\}$. Since the $f_{i}^{(\alpha+1)}$ are all regular for $i \in\{1, \ldots, m\}$, we can let $g_{i}, h$ be positive linear functionals so that for $i \in\{1, \ldots, m\}$ we have

$$
f_{i}^{(\alpha+1)}=g_{i}-h \text {. }
$$

Due to compactness of the set $\mathcal{A}_{e}^{m+1}$ we can pick $\left(y_{1}^{*}, \ldots, y_{m+1}^{*}\right) \in \mathcal{A}_{e}^{m}$ in such a way that the bound

$$
\begin{aligned}
& \sum_{i=1}^{m}\left(f_{i}^{(1)}\left(y_{i}^{*}\right), \ldots, f_{i}^{(\alpha)}\left(y_{i}^{*}\right), g_{i}\left(y_{i}^{*}\right)\right)+(0, \ldots, 0, h)\left(y_{m+1}^{*}\right) \\
& \quad \geq \sum_{i=1}^{m}\left(f_{i}^{(1)}\left(x_{i}\right), \ldots, f_{i}^{(\alpha)}\left(x_{i}\right), g_{i}\left(x_{i}\right)\right)+(0, \ldots, 0, h)\left(x_{m+1}\right)
\end{aligned}
$$

holds for each $\left(x_{1}, x_{2}, \ldots, x_{m+1}\right) \in \mathcal{A}_{e}^{m+1}$. By positivity of $(0, \ldots, 0, h)$ we can assume without loss of generality that $\sum_{j=1}^{m+1} y_{j}^{*}=e$ so that $y_{m+1}^{*}=e-\sum_{j=1}^{m} y_{j}^{*}$. Then we obtain that the set of functions $\left\{\left(f_{i}^{(1)}, \ldots, f_{i}^{(\alpha)}, g_{i}\right): i \in\{1, \ldots, m\}\right\} \cup$ $\{(0, \ldots, 0, h)\}$ satisfies the assumptions we made in the first half of this proof. Hence we get by our previous results

$$
\begin{aligned}
g(e)= & \left(\bigvee_{i=1}^{m}\left(f_{i}^{(1)}, \ldots, f_{i}^{(\alpha)}, g_{i}^{(\alpha+1)}-h\right)\right)^{+}(e) \\
= & \left(\left(\bigvee_{i=1}^{m}\left(\left(f_{i}^{(1)}, \ldots, f_{i}^{(\alpha)}, g_{i}\right)-(0, \ldots, 0, h)\right)\right)\right. \\
& \vee((0, \ldots, 0, h)-(0, \ldots, 0, h)))(e) \\
= & \left(\bigvee_{i=1}^{m}\left(f_{i}^{(1)}, \ldots, f_{i}^{(\alpha)}, g_{i}\right) \vee(0, \ldots, h)\right)(e)-(0, \ldots, 0, h)(e) \\
= & \sum_{i=1}^{m}\left(f_{i}^{(1)}\left(y_{i}^{*}\right), \ldots, f_{i}^{(\alpha)}\left(y_{i}^{*}\right), g_{i}\left(y_{i}^{*}\right)\right) \\
& +\left(0, \ldots, 0, h\left(e-\sum_{i=1}^{m} y_{i}^{*}\right)\right)-(0, \ldots, 0, h(e)) \\
= & \sum_{i=1}^{m}\left(f_{i}^{(1)}\left(y_{i}^{*}\right), \ldots, f_{i}^{(\alpha)}\left(y_{i}^{*}\right), g_{i}\left(y_{i}^{*}\right)-h\left(y_{i}^{*}\right)\right) \\
& +(0, \ldots, 0, h(e)-h(e)) \\
= & \sum_{i=1}^{m}\left(f_{i}^{(1)}\left(y_{i}^{*}\right), \ldots, f_{i}^{(\alpha)}\left(y_{i}^{*}\right), f_{i}^{(\alpha+1)}\left(y_{i}^{*}\right)\right) \\
= & \sum_{i=1}^{m} f_{i}\left(y_{i}^{*}\right) \\
\leq & \sum_{i=1}^{m} f_{i}\left(x_{i}^{*}\right)
\end{aligned}
$$

by our choice of $x_{1}^{*}, \ldots, x_{m}^{*}$. Since in the very first part of the proof we saw that $g(e) \geq \sum_{i=1}^{m} f_{i}\left(x_{i}^{*}\right)$ we must have $g(e)=\sum_{i=1}^{m} f_{i}\left(x_{i}^{*}\right)$ and that finishes the proof of part (i).

Proof of (ii) The first statement follows for the same reason as in part (i). So pick $\left(x_{1}^{*}, \ldots, x_{m}^{*}\right) \in \mathcal{F}_{e}^{m}$ such that $\sum_{i=1}^{m} f_{i}\left(x_{i}^{*}\right) \geq \sum_{i=1}^{m} f_{i}\left(x_{i}\right)$ for each $\left(x_{1}, x_{2}, \ldots, x_{m}\right) \in \mathcal{F}_{e}^{m}$. First note since $\left(x_{1}^{*}, \ldots, x_{m}^{*}\right) \in \mathcal{F}_{e}^{m}$ that in fact $x_{1}^{*}=$ $e-\sum_{i=2}^{m} x_{i}^{*}$. Upon introducing $g=\bigvee_{i=1}^{m} f_{i}$, we start by observing that $g-f_{1}=$ $\left[\bigvee_{i=2}^{m}\left(f_{i}-f_{1}\right)\right]^{+}$, together with the estimate

$$
\begin{aligned}
\sum_{i=2}^{m}\left(f_{i}-f_{1}\right)\left(x_{i}^{*}\right) & =\sum_{i=1}^{m} f_{i}\left(x_{i}^{*}\right)-f_{1}(e) \\
& \geq \sum_{i=2}^{m} f_{i}\left(x_{i}\right)+f_{1}\left(e-\sum_{i=2}^{m} x_{i}\right)-f_{1}(e) \\
& =\sum_{i=2}^{m}\left(f_{i}-f_{1}\right)\left(x_{i}\right)
\end{aligned}
$$


for any $\left(x_{2}, \ldots, x_{m}\right) \in \mathcal{A}_{e}^{m-1}$, since if $\left(x_{2}, \ldots, x_{m}\right) \in \mathcal{A}_{e}^{m-1}$ then $(e-$ $\left.\sum_{i=2}^{m} x_{i}, x_{2}, \ldots, x_{m}\right) \in \mathcal{F}_{e}^{m}$. Therefore by part (i) we have that

$$
\left(g-f_{1}\right)(e)=\sum_{i=2}^{m}\left(f_{i}-f_{1}\right)\left(x_{i}^{*}\right)
$$

In particular, we obtain

$$
\begin{aligned}
g(e) & =f_{1}\left(e-\sum_{i=2}^{m} x_{i}^{*}\right)+\sum_{i=2}^{m} f_{i}\left(x_{i}^{*}\right) \\
& =\sum_{i=1}^{m} f_{i}\left(x_{i}^{*}\right)
\end{aligned}
$$

which is the desired formula.

Proof of (iii) Now fix an arbitrary $x \in L^{+}$and then select an order unit $e \in L$ which has $x \leq e$.

For each $0<\beta<1$, set $x_{\beta}=\beta x+(1-\beta) e$. Clearly, each $x_{\beta}$ is an order unit and $0 \leq x_{\beta} \leq e$ holds for each $0<\beta<1$. We consider the index set $(0,1)$ directed by the increasing order relation $\succeq$, i.e. $\beta \succeq \gamma$ in $(0,1)$ if and only if $\beta \geq \gamma$. It is clear that we have the limit $x_{\beta} \rightarrow x$. Since the set $\mathcal{F}_{e}^{m}$ is compact, the continuous function $\mathcal{F}_{e}^{m} \rightarrow M,\left(x_{1}, \ldots, x_{m}\right) \mapsto \sum_{i=1}^{m} f_{i}\left(x_{i}\right)$ attains its maximum. By part (ii), we know that $g$ satisfies the Riesz-Kantorovich formula for each order unit. Therefore, by our assumption on the space $M$ and the compactness of $\mathcal{F}_{e_{\beta}}^{m}$, for each $0<\beta<1$ there exists some $\left(z_{1}^{\beta}, \ldots, z_{m}^{\beta}\right) \in L_{+}^{m}$ such that $\sum_{i=1}^{m} z_{i}^{\beta}=x_{\beta}$ and

$$
\begin{aligned}
\beta g(x)+(1-\beta) g(e) & =g\left(x_{\beta}\right) \\
& =\sum_{i=1}^{m} f_{i}\left(z_{i}^{\beta}\right) .
\end{aligned}
$$

Since $z_{i}^{\beta} \in[0, e]$ for each $\beta \in(0,1)$ and each $i \in\{1, \ldots, m\}$ and the order interval $[0, e]$ is compact, there exists a subnet of $\left\{\left(z_{1}^{\beta}, \ldots, z_{m}^{\beta}\right)\right\}$ (which without loss of generality we also denote by $\left.\left\{\left(z_{1}^{\beta}, \ldots, z_{m}^{\beta}\right)\right\}\right)$ in such a way that $z_{i}^{\beta} \rightarrow z_{i}$ for some $z_{i} \in[0, e]$. From $x_{\beta}-z_{i}^{\beta} \in[0, e]$ for each $\beta$ and the closedness of $[0, e]$, we see that $0 \leq x-z_{i} \leq e$. So $0 \leq z_{i} \leq x$ and $\sum_{i=1}^{m} z_{i}=x$. Finally, letting $\beta \rightarrow 1$ in Eq. (3.46) it follows from the continuity of each $f_{i}$ that

$$
g(x)=\sum_{i=1}^{m} f_{i}\left(z_{i}\right)
$$

Since we already know that $g(x) \geq \sum_{i=1}^{m} f_{i}\left(y_{i}\right)$ for all $\left(y_{1}, \ldots, y_{m}\right) \in \mathcal{F}_{x}^{m}$, it follows that $\sup \left\{\sum_{i=1}^{m} f_{i}\left(y_{i}\right):\left(y_{1}, \ldots, y_{m}\right) \in \mathcal{F}_{x}^{m}\right\}=g(x)$. So the proof of this induction step is finished.

Finally, let $\alpha$ be a limit ordinal and assume that the theorem holds for all image spaces $\prod_{1 \leq j \leq \beta}$ for $\beta<\alpha$. Set $M=\prod_{1 \leq j \leq \alpha} \mathbb{R}$ and let $f_{1}, f_{2}, \ldots, f_{m}$ be continuous, linear maps from $L$ to $M$ such that $f_{i}^{(j)}$ is regular for all $i \in\{1, \ldots, m\}$ and all ordinals $j$ with $1 \leq j \leq \alpha$ and such that the supremum $g:=\bigvee_{i=1}^{m} f_{i}$ exists in $L^{\sim}(L, M)$. 
Let $x \in L_{+}$. Let $\beta<\alpha$. Since we know from Lemma 2.3 that $\bigvee_{i=1}^{m}\left(f_{i}^{(1), \ldots,(\beta)}\right)=$ $\bigvee_{i=1}^{m} f_{i}^{(1), \ldots,(\beta)}$ we can use the induction hypothesis to obtain that

$$
g(x)^{(1), \ldots,(\beta)}=\sup \left\{\sum_{i=1}^{m} f_{i}\left(x_{i}\right)^{(1), \ldots,(\beta)}:\left(x_{1}, \ldots, x_{m}\right) \in \mathcal{F}_{x}^{m}\right\} .
$$

Since we use the lexicographic ordering on $M$, we know that $\sup \left\{\sum_{i=1}^{m} f_{i}\left(x_{i}\right)\right.$ : $\left.\left(x_{1}, \ldots, x_{m}\right) \in \mathcal{F}_{x}^{m}\right\}$ exists in $M$ and that we have the identity

$$
\begin{aligned}
g(x)^{(1), \ldots,(\beta)} & =\sup \left\{\sum_{i=1}^{m} f_{i}\left(x_{i}\right)^{(1), \ldots,(\beta)}:\left(x_{1}, \ldots, x_{m}\right) \in \mathcal{F}_{x}^{m}\right\} \\
& =\sup \left\{\sum_{i=1}^{m} f_{i}\left(x_{i}\right):\left(x_{1}, \ldots, x_{m}\right) \in \mathcal{F}_{x}^{m}\right\}^{(1), \ldots,(\beta)}
\end{aligned}
$$

Since this identity holds for all $\beta<\alpha$, we immediately obtain

$$
g(x)=\sup \left\{\sum_{i=1}^{m} f_{i}\left(x_{i}\right):\left(x_{1}, \ldots, x_{m}\right) \in \mathcal{F}_{x}^{m}\right\}
$$

and the proof is finished.

Remark 3.2 From the proof of this theorem it becomes clear why we used the lexicographic ordering instead of the componentwise ordering. One of the key properties of the lexicographic ordering is that each compact set has an order-maximum, which we use extensively. This is not true for the componentwise ordering as can be seen from the following example. Let $f: \mathbb{R} \rightarrow \mathbb{R}^{2}, \lambda \mapsto \lambda(-1,1)$ and $g: \mathbb{R} \rightarrow \mathbb{R}^{2}, \lambda \mapsto 0$. Then we see that $\{f(1-y)+g(y): 0 \leq y \leq 1\}=\{\lambda(-1,1): 0 \leq \lambda \leq 1\}$. This set does not have an order-maximum, since its order-supremum equals $(0,1)$, which is not an element of this set. Since sets of the form $\{f(1-y)+g(y): 0 \leq y \leq 1\}$ are precisely the sets for which we need an order-maximum to exist, there is no hope to prove a similar statement for the componentwise ordering in the same way we did for the lexicographic ordering. Note, however, that [6, Lemma 3.1] and [6, Lemma 3.2], which form the base for steps (i) and (ii) in the proof above, can easily be proven for the componentwise ordering.

The following corollary might give a more general solution to the RieszKantorovich problem than before. It is concerned with the situation of Theorem 3.1, but then for spaces without a strong order unit. It is based on [6, Lemma 3.6].

Corollary 3.3 Let L be a partially ordered vector spaces with a Hausdorff topology for which the order-intervals are compact. Let $\alpha \geq 1$ be an ordinal number and let $M=\prod_{1 \leq j \leq \alpha} \mathbb{R}$ equipped with the lexicographic ordering. Then the following statements are equivalent.

1. There is a pair $f, g \in L^{r}(L, M)$ of continuous maps such that $f^{(j)}$ and $g^{(j)}$ are regular for all $1 \leq j \leq \alpha$, such that $f \vee g$ exists in $L^{\sim}(L, M)$, but does not satisfy the Riesz-Kantorovich formula for all $x \in L_{+}$.

2. There is a pair $f, g \in L^{r}(L, M)$ of continuous maps and a point $x \in L_{+}$such that $f^{(j)}$ and $g^{(j)}$ are regular for all $1 \leq j \leq \alpha$, such that $f \vee g$ exists in $L^{\sim}(L, M)$, but the restriction $\left.(f \vee g)\right|_{L_{x}}$ is not the supremum of $\left.f\right|_{L_{x}}$ and $\left.g\right|_{L_{x}}$ in $L^{\sim}\left(L_{x}, M\right)$. 
Proof Assume that (1) holds and let $f, g \in L^{r}(L, M)$ be a pair of continuous maps such that $f^{(j)}$ and $g^{(j)}$ are regular for all $1 \leq j \leq \alpha$, such that $f \vee g$ exists in $L^{\sim}(L, M)$, but does not satisfy the Riesz-Kantorovich formula. Then we can let $x \in L_{+}$such that

$$
f \vee g(x)>\sup \{f(y)+g(x-y): 0 \leq y \leq x\} .
$$

Consider the space $L_{x}$. As mentioned earlier we get that $x$ is a strong order unit for $L_{x}$. Seeking a contradiction, we assume that $\left.\left.f\right|_{L_{x}} \vee g\right|_{L_{x}}$ exists in $L^{\sim}\left(L_{x}, M\right)$ and that

$$
\left.(f \vee g)\right|_{L_{x}}=\left.\left.f\right|_{L_{x}} \vee g\right|_{L_{x}} .
$$

Then by Theorem 3.1, which we can apply since $\left.f\right|_{L_{x}}$ and $\left.g\right|_{L_{x}}$ satisfy the conditions of Theorem 3.1 and the space $L_{x}$ has a strong order unit, we obtain that

$$
\begin{aligned}
\left(\left.\left.f\right|_{L_{x}} \vee g\right|_{L_{x}}\right)(x) & =\sup \left\{\left.f\right|_{L_{x}}(y)+\left.g\right|_{L_{x}}(x-y): y \in L_{x}, 0 \leq y \leq x\right\} \\
& =\sup \left\{\left.f\right|_{L_{x}}(y)+\left.g\right|_{L_{x}}(x-y): y \in L, 0 \leq y \leq x\right\} \\
& <(f \vee g)(x) \\
& =\left(\left.\left.f\right|_{L_{x}} \vee g\right|_{L_{x}}\right)(x),
\end{aligned}
$$

where we used the definition of $L_{x}$ and the assumptions. This yields a contradiction. Therefore (2) holds and we obtain the implication $(1) \Rightarrow(2)$.

Conversely, suppose that (2) is true. Let $f, g \in L^{r}(L, M)$ be a pair of continuous maps and pick $x \in L_{+}$in such a way that $f^{(j)}$ and $g^{(j)}$ are regular for all $1 \leq j \leq \alpha$, such that $f \vee g$ exists in $L^{\sim}(L, M)$, but the restriction $\left.(f \vee g)\right|_{L_{x}}$ is not the supremum of $\left.f\right|_{L_{x}}$ and $\left.g\right|_{L_{x}}$ in $L^{\sim}\left(L_{x}, V\right)$. Again we argue by contradiction. Assume that $f \vee g$ satisfies the Riesz-Kantorovich formula for all $z \in L_{+}$. Pick $h \in L^{\sim}\left(L_{x}, M\right)$ such that $h \geq\left. f\right|_{L_{x}}$ and $h \geq\left. g\right|_{L_{x}}$ and fix $z \in L_{x}^{+}$and $0 \leq y \leq z$. Then we obtain since $y, z-y \in L_{x}$ that

$$
\begin{aligned}
h(z) & =h(y)+h(z-y) \\
& \geq\left. f\right|_{L_{x}}(y)+\left.g\right|_{L_{x}}(z-y) .
\end{aligned}
$$

Therefore we must have that

$$
\begin{aligned}
h(z) & \geq \sup \left\{\left.f\right|_{L_{x}}(y)+\left.g\right|_{L_{x}}(z-y): 0 \leq y \leq z\right\} \\
& =\sup \{f(y)+g(z-y): 0 \leq y \leq z\} \\
& =(f \vee g)(z) \\
& =\left.(f \vee g)\right|_{L_{x}}(z),
\end{aligned}
$$

since $f \vee g$ satisfies the Riesz-Kantorovich formula. Therefore we see that $h \geq$ $\left.(f \vee g)\right|_{L_{x}}$. Furthermore we obtain for $z \in L_{+}$that 


$$
\begin{aligned}
\left.(f \vee g)\right|_{L_{x}}(z) & =(f \vee g)(z) \\
& \geq f(z) \\
& =\left.f\right|_{L_{x}}(z)
\end{aligned}
$$

and similarly $\left.(f \vee g)\right|_{L_{x}}(z) \geq\left. g\right|_{L_{x}}(z)$. This yields $\left.(f \vee g)\right|_{L_{x}} \geq\left.\left. f\right|_{L_{x}} \vee g\right|_{L_{x}}$. Combining these two statements gives that $\left.(f \vee g)\right|_{L_{x}}=\left.\left.f\right|_{L_{x}} \vee g\right|_{L_{x}}$ which gives a contradiction to our assumption. Therefore (1) must hold and we obtain (2) $\Rightarrow$ (1).

Since the componentwise ordering on products of $\mathbb{R}$ is used more often than the lexicographic ordering, it is of course of much interest to prove a statement similar to Theorem 3.1 for the componentwise ordering. As stated before it is not difficult to show an equivalent for [6, Lemma 3.1] and [6, Lemma 3.2] for this case, but the problem lies in the fact that a compact set no longer needs to have an order-maximum, so the proof of [6, Theorem 3.3] cannot be adapted easily.

It is remarkable to note the following: since a product of at least two times the real line with the lexicographic ordering is not even Archimedean, it is as a Riesz space in some sense as far away as possible from being Dedekind complete, since Dedekind completeness implies that a space is Archimedean. So in fact, we now know that the Riesz-Kantorovich formula holds for the most 'extreme' image spaces: the Dedekind complete ones on one hand and the ones with the lexicographic ordering on the other hand. So even though one likes to think of the Dedekind complete spaces as 'better' spaces than other Riesz spaces, in this case that is not entirely justified, especially since the conditions on the domain space are a lot weaker for the lexicographic ordering than for the Dedekind complete case. So in proving the Riesz-Kantorovich formula for the general case, it turns out that the more extreme special cases are in fact the easiest to consider.

Open Access This article is distributed under the terms of the Creative Commons Attribution 4.0 International License (http://creativecommons.org/licenses/by/4.0/), which permits unrestricted use, distribution, and reproduction in any medium, provided you give appropriate credit to the original author(s) and the source, provide a link to the Creative Commons license, and indicate if changes were made.

\section{References}

1. Abramovich, Y.A., Wickstead, A.W.: Regular operators from and into small Riesz spaces. Indag. Math. N.S. 2, 257-274 (1991)

2. Abramovich, Y.A., Wickstead, A.W.: The regularity of order bounded operators into $C(K)$, II. Q. J. Math. Oxf. Ser (2) 44, 257-270 (1993)

3. Aliprantis, C.D., Border, K.C.: Infinite Dimensional Analysis: A Hitchhikers Guide, 3rd edn. Springer, New York (1999)

4. Aliprantis, C.D., Burkinshaw, O.: Positive Operators, 119th edn. Springer Science \& Business Media, Berlin (2006)

5. Aliprantis, C.D., Tourky, R.: The super order dual of an ordered vector space and the Riesz-Kantorovich formula. Trans. Am. Math. Soc. 354, 2055-2077 (2002)

6. Aliprantis, C.D., Tourky, R., Yannelis, N.C.: The Riesz-Kantorovich formula and general equilibrium theory. J. Math. Econ. 34, 55-76 (2000)

7. De Jonge, E., Van Rooij, A.C.M.: Introduction to Riesz Spaces. Mathematical Centre, Amsterdam (1977) 
8. Kantorovich, L.V.: Concerning the general theory of operations in partially ordered spaces. Dokl. Akad. Nauk SSSR 1, 271-274 (1936) (in Russian)

9. Luxemburg, W.A.J., Zaanen, A.C.: Riesz Spaces, vol. I. North-Holland Publishing Company, Amsterdam (1971)

10. Riesz, M.: Sur quelques notions fondamentals dans la theorie générale des opérations linéaires. Ann. Math. 41, 174-206 (1940)

11. Schouten, W.M.: The Riesz-Kantorovich formula for lexicographically ordered spaces. Master Thesis, Leiden University (2016). http://www.math.leidenuniv.nl/scripties/MasterSchouten.pdf

12. van Rooij, A.C.M.: When do the regular operators between two Riesz spaces form a vector lattice? Indag. Math. 47, 95-98 (1985) 